Radial and Nonradial Pulsations as Probes of Stellar Physics

ASP Conference Series, Vol. 259, 2002

C. Aerts, T.R. Bedding, \& J. Christensen-Dalsgaard, eds.

\title{
On the Theory of Oscillations of Rapidly Rotating Stars
}

\author{
M. Rieutord \\ Observatoire Midi-Pyrénées, 14 av. Edouard Belin F-31400 Toulouse, \\ and Institut Universitaire de France \\ F. Lignières \\ Observatoire Midi-Pyrénées, 57 av. d'Azereix, BP 826, F-65008 Tarbes
}

\begin{abstract}
We present the current understanding of the dynamical aspects of the oscillations of rapidly rotating stars.
\end{abstract}

In this contribution, we first review the recent progress concerning the role of the Coriolis force when its amplitude is such that perturbative approaches are inappropriate. We then discuss the problems raised by the existence of the centrifugal acceleration and show that, although of second order, it should affect quite strongly the spectrum of acoustic modes of rapidly rotating stars.

The role of the Coriolis force on the oscillations of a star is twofold: it perturbs already existing modes (like acoustic, gravity or Alfven modes) and introduces new modes, namely inertial modes, for which it is the restoring force. In radiative zones of stars, $N_{\max }>2 \Omega$ and we usually have to deal with gravity modes perturbed by rotation and gravito-inertial modes, while in convective zones $N_{\max } \sim 0$ implies that we are left with pure inertial modes. Acoustic modes, being in the high frequency range, are usually only slightly perturbed by the Coriolis force. Pure inertial modes, being constrained to convective zones, should not be expected to reach large amplitudes because their excitation is stochastic. On the other hand, gravity modes excited by an instability $(\gamma, \kappa, \ldots)$ can reach large amplitudes and, if their frequency is close to $2 \Omega$, be strongly perturbed by rotation; an example is $\gamma$ Doradus stars (Dintrans \& Rieutord, 2000).

The strong effect of the Coriolis force is of mathematical origin. To be fully appreciated, it is convenient to focus on an incompressible inviscid fluid contained in a spherical shell which mimics the convective or radiative zone of a star. Such a configuration only possesses inertial modes but this is sufficient for our purpose. The pressure perturbations associated with inertial modes satisfy Poincaré's equation (see Greenspan, 1969), namely $\Delta p-\left(\frac{2 \Omega}{\omega}\right)^{2} \frac{\partial^{2} p}{\partial z^{2}}=0$, where $\omega$ is the frequency of the mode. It can be shown that $|\omega| \leq 2 \Omega$ which makes Poincaré's equation of hyperbolic type. However, fluid motions satisfy the boundary condition $\vec{v} \cdot \vec{n}=0$, where $\vec{n}$ is a vector normal to the boundary; therefore, the problem is mathematically ill-posed, since a hyperpolic equation requires initial conditions rather than boundary conditions. This mathematical property has major consequences on the form of eigenmodes of a rotating fluid: in general, there are no smooth solutions and no eigenmode exists for an inviscid fluid. In other words, if we consider the (damped) eigenmodes of the associated 
viscous problem, their kinetic energy tends to infinity as viscosity decreases to zero (Rieutord et al., 2000, 2001). Such a property is not specific to inertial modes and is shared also by gravity modes. Hence, these latter modes also suffer from these singularities in general (Rieutord \& Noui, 1999) but, in the case of a non-rotating star, the spherical symmetry of the configuration permits the separation of variables and thus removes the singular solutions; however, as soon as separability disappears singularities come back.

To conclude the case of Coriolis force, let us emphasize that it deeply modifies the shape of eigenmodes in the range $\omega \lesssim 2 \Omega$ and that diffusion is a necessary ingredient to properly compute these modes. Adiabatic calculations may still give 'good looking' results, especially if the numerical method is dissipative, but they will often converge to spurious modes often betrayed by a slow convergence when grid resolution is increased.

The centrifugal force is proportional to $\Omega^{2}$ whereas the Coriolis force is linear with $\Omega$. One thus expects that centrifugal effects are less important than those of the Coriolis force. Unfortunately, this is not true for rapidly rotating stars. The first effect of the centrifugal force is to modify the equilibrium shape of the star by inducing a polar flattening $\varepsilon$. So very basically, the fluid's container is changed. This change is very important as it affects mostly the outer layers which contain most of the amplitude of observable modes. If $R$ is the star radius, all modes whose wavelength is of order $\varepsilon R$ will be sensitive to the ellipsoidal shape of the container; the period of such modes is $\varepsilon R / c_{s}$ which is $\sim 25 \mathrm{mn}$ for a star with $\mathrm{M}=2 \mathrm{M}_{\odot}$ and $\mathrm{R}=2 \mathrm{R}_{\odot}$ rotating at $150 \mathrm{~km} \mathrm{~s}^{-1}$ when $c_{s} \sim 100 \mathrm{~km} \mathrm{~s}^{-1}$; these numbers are typical of $\delta$ Scuti stars. The second effect comes from the impossibility of having radiative zones at rest when the fluid is rotating (Von Zeipel theorem). These motions defining the quasi-steady state of the star are little known (turbulent or not ?); by the implied mixing, they may be effective during the whole evolution of the stars.

The centrifugal force has an influence which is presently not clear. Its first role in changing the shape of the star is currently under study (Lignières et al., 2001) and is suspected to have a great influence on acoustic modes in rapidly rotating stars like $\delta$ Scuti stars. Its second, but more subtile, role is its long-term influence during the evolution by the mixing processes it induces. These effects need to be appreciated through simulations of the evolution of a rotating star using 2D models accounting for the transport mechanisms.

Acknowledgments. We are much indebted to Lorenzo Valdettaro for his help in the elaboration of the numerical solutions.

\section{References}

Dintrans, B. \& Rieutord, M. 2000, A\&A, 354, 86

Greenspan, H. 1969, The theory of rotating fluids, (Cambrige University Press) Lignières, F., Rieutord, M., \& Valdettaro, L. 2001, Semaine de l'Astrophysique Francaise, Edts, SF2A, (EdP-Sciences, Conf. Series)

Rieutord, M. \& Noui, K. 1999, Euro. Phys. J. B, 9, 731

Rieutord, M., Georgeot, B., \& Valdettaro, L. 2000, PRL, 85, 4277

Rieutord, M., Georgeot, B., \& Valdettaro, L. 2001, JFM, 435, 103 